\title{
Investigating the relationship between auditor's opinion and stock return in the companies listed at Tehran stock exchange market
}

\author{
Ali Akbar Farzinfar*
}

Corresponding Author, Accounting \& Management Department, Kashan Branch, Islamic Azad University, Kashan, Iran, Iran

\section{H R O N I C L E}

Article history:

Received June 28, 2012

Received in revised format

26 October 2012

Accepted 10 November 2012

Available online

November 122012

Keywords:

Auditing

Auditor

Capital

Working Capital

Auditing Standards

Stock shareholder

Stock return \begin{abstract}
A B S T R A C T
This research investigates the relationship between auditor's opinion and stock return in the companies listed at Tehran stock exchange market. In this study, all required data are collected from aware shareholders and provide a sampling of 130 questionnaires, the data collected over the period 2010-2011 using test methods such as computer software, data analysis and statistical methods to answer research questions. According to research result through questionnaires and tests, there is a significant relationship between stock returns and the auditor's opinion, in fact, for aware shareholders of the company the auditor's opinion has a special message. This message is based on the results of study hypothesis in comparison with previous research with regard to changes in the questionnaire and provides assumptions that are more detailed. The first finding is that unqualified audit report has a positive impact on stock returns of companies with a medium to low degree. Adverse audit report has a negative impact on stock returns of companies with medium to high degree. Disclaimer of audit report has a negative impact on stock returns of companies with medium to high degree. Finally, qualifying paragraphs, which modified the items of income statement items have more impact in comparison with qualifying paragraphs which modified the Balance Sheet items.
\end{abstract}

\section{Introduction}

The financial statements audited by independent auditors are appropriate instruments to transfer reliable information. A competent auditor audits according to the auditing standards to ensure that the items set forth in the financial statements have been prepared as per accounting standards. That means an auditor validates the claims made by any other person in form of financial statements, and increases the reliability of the information used for making economic decisions. Among all institutions of a country, economic institution has a special importance and status. Capital market is one of the most important subsections of economy. In these markets, savers provide their surplus cash to those ones requiring liquidity to continue the business of their business enterprises.

\footnotetext{
${ }^{*}$ Corresponding author.

E-mail addresses: farzinfar_47@yahoo.com (A. A. Farzinfar) 
It is understood that the increase in the efficiency of such markets helps transfer cash more conveniently, enhances the power of business enterprises in performing their activities, and finally leads to the economic upturn. In such a sound economy and capital markets, stock exchange is of considerable importance. In the stock exchange, the capitals of business enterprises are negotiated in form of stocks or securities, and cash flow is administered based on predetermined mechanisms. In case, the stock exchange finds its ideal status in the economy of a country, and plays its role well, it accelerates to the economic upturns and development of that country. In the present economic conditions of Iran, it is required inevitably to pay enough attention to the stock exchange and all institutions and individuals involved in the process of stock exchange's activities should perform their tasks and duties in an appropriate manner to pave the way for achieving ideal economic growth and transfer from a traditional economic system to the modern one.

The most important informing instrument in this market is the financial information with required quality specifications, from which reliability is of importance. Financial information is reliable when financial events and other financial transactions are measured fairly, and as a result, the users of such financial information can rely on them as the reliability of such information is confirmed by an independent, fair, and competent person. In the present economic system, financial statements are audited by independent auditors for voicing their opinion through auditor's report. It seems that the best information source used to determine the yields of a business enterprise is its financial statements provided by the management of the enterprise together with the independent auditor's report. These sources are used for making right decisions based on the provided financial statements. Taking into this fact, the present research aims to study the significance of the opinions expressed in the auditor's reports and its effects on the stock return of the companies listed on Tehran Stock Exchange.

During the past three decades, various researches have been conducted to survey the economic effects of economic opinions of auditors. In the USA, the researches of Elliott (1982), Louder et al. (1992), Chen and Church (1992, 1996), Dopuch et al. (1986), and Davis (1982) are examples of the studies conducted in this regard. Elliott (1982) studied the abnormal returns of 145 companies in two groups based on "subject to" qualification paragraphs and going-concern opinion doubting the continued existence of companies for the period over the period 1973-1978. The companies with qualified reports had an abnormally negative return within 45 weeks before the public issuance of the auditor's report, while no abnormal negative return was observed one week after its issuance. The results of the research of Louder et al. (1992) showed that if market expectations are directed appropriately, "subject to" qualifications provides suitable information.

The studies of Chen and Church (1996) on the association between the going-concern qualifications and market reactions to the bankrupted companies showed that the companies receiving goingconcern opinion had negative cumulative return less than that of the companies with unqualified opinion during their activities period after bankruptcy. The results showed that going-concern opinion contains valuable information.

Dopuch et al. (1986) studied the impacts of "subject to" audit opinion paragraphs on the stock price of 114 sampled companies. The data of public issuance is the very date of the issuance of the auditor's report in gazettes. The results of this research show that the stock of the companies receiving "subject to" paragraphs in their audit opinion have abnormal negative return for both first and second time of the study. Davis (1982) studied in his research the changes in the stock price of the companies that were similar in terms of industry, error coefficient of profit forecast, and systematic risks. These companies were classified into two groups of receiving qualified and unqualified opinions. The comparison of the changes in the stock price of these two groups at the time of public issuance reflected no significant difference. The reason of such differences in the results might be due to the methodology of researches (different data of public issuance at the same time of the issuance of auditor's report). 
Khatami (1997) studied and tested the impacts of audit qualifications on stock price and the analysis of annual financial statements. In this research, the auditor's reports of the companies listed on Tehran Stock Exchange were studied and it was concluded that the reactions of the stockholders and other investors were positive to the results of the companies with less qualifications in comparison to the companies with more qualifications. This result is consistent with those obtained by the researchers, who conducted their researches in other stock exchanges.

Hajiha (2012) identified the critical influencing factors on risks proposed in Audit Risk Model (ARM), in Iranian audit environment of Iran by using 60 audit partners and managers. The panel included two equally divided groups, one from audit organization, a governmental organization, and the other from private audit firms. She employed two rounds of Delphi and 58 critical risk factors obtained from auditing literature and Iranian auditing standards and present them to the experts. There were 43 factors categorized as important factors to evaluate the risks in ARM. The results specified the most important factors, which were in inherent risk factors. She made a comparison with a similar study in Taiwan and differences indicated that in professional judgment issues like risk assessment, the consideration of particular culture and environment could contribute to enhance the precision of assessments, especially in assessing control risk factors.

\section{Research main problem and hypotheses}

The main question of this research is to consider the impact of auditor's opinion on the stock price for the listed companies in Tehran Stock Exchange.

1- Unqualified audit report has a positive effect on the stock return of companies.

2- Adverse audit report is effective in the stock return of companies.

3- Disclaimer of audit report is effective in the stock return of companies.

4- The qualification paragraphs leading to the modification and adjustment of gain and loss items are more effective in the stock return of companies than balance sheet items are.

\subsection{General Research Method}

Relevant theoretical frameworks and standards have been applied, and library method has been used to recognize variables and test the effective factors and hypotheses. To collect the required data, a questionnaire has been prepared and distributed among the respondents for the test the hypotheses. The results obtained from the sample of companies are generalized to the total statistical population. For the purpose of this research, Excel application software is used to process the data and analyze them by SPSS statistical program.

\subsection{Statistical Population and Sample}

The statistical population of this research consists of investors, analysts, and shareholders. Out of the total members of the statistical population, a sample including senior auditors and financial managers of the studied companies is selected. These members are selected as they are fully informed of the definitions provided in the questionnaire. The data collected by the questionnaire confirms this fact.

The criteria used for taking a sample from the population are as follows:

- The sample must be representative of the statistical population and have the specifications as regards the subject of this research.

- All members of the statistical population must have the same chance of selection.

- The selected members of the sample must be acquainted with shares and audit reports. 
Considering that the information used in this research is of qualitative type, and as the variance of the society is unknown to us, we use the maximum dispersion of random variable. To determine the minimum size of the sample, we assume variance equal to $\sigma^{2}=0.25$.

$n=\frac{\left(Z_{\alpha / 2}\right)^{2} \sigma^{2}}{\varepsilon^{2}}$,

where, $\alpha$ is the acceptable level of non-confidence. In other words, $1-\alpha$ is the acceptable level of confidence. In other words, $1-\alpha$ is the level of confidence, and as the distribution is bilateral, $\alpha$ is divided by 2 , and $\mathrm{z}$ is the numerical value of the quantity in the numerical tables with normal standard distribution for the confidence level of $1-\alpha / 2$. Moreover, as $n$ is unknown, the error of estimation $(\varepsilon)$ has been calculated based on the acceptable domain. Therefore, the size of the sample is as follows:

$n=\frac{\left(Z_{\alpha / 2}\right)^{2} \sigma^{2}}{\varepsilon^{2}}=\frac{(1.96)^{2} \times 0.25}{0.1^{2}}=96$.

For the purpose of this research, 130 questionnaires have been distributed among the auditors and financial managers, who completed 100 questionnaires. As the members of the sample are more than 30 , it can be concluded that the population is distributed normally. Therefore, the results obtained from the sample can be generalized to the total population. To test the hypotheses of this research, the required data are weighted based on Likert method. According to this method, the respondents are requested to determine the effects of each factor on stock return using a continuum divided into five parts. For the statistical test of the hypotheses, it is indeed required to convert the qualitative data into quantitative ones. Therefore, several questions have been provided for each hypothesis, and they are weighted using the five-point Likert scale (strongly high, high, moderate, low, and strongly low). To convert these qualitative items into quantitative ones, they are ranked from 1 to 5.

Validity and reliability are two concepts required for the measurement of the suitability and effectiveness of the research and methods of data collection. Validity is the rational relation between the test questions and the tested subject. Reliability of a questionnaire means that it can perform its required functions under stated conditions for a specified period. Unreliability of a questionnaire may be due to the unclear questions, their irrelevance to hypotheses, etc. One of the most popular criteria used for the assessment of reliability is Cronbach's Alpha, which is defined as follows:

$\alpha=\frac{K}{K-1}\left[1-\frac{\sum s_{i}^{2}}{s_{\text {sum }}^{2}}\right]$,

where, $s_{i}^{2}$ is the variance of the $i^{\text {th }}$ question, $s_{\text {sum }}^{2}$ is the variance of total questions, and $k$ is the number of the questions of the questionnaire. If the value of alpha approaches more to 1 the reliability increases accordingly. In contrast, the low value of alpha indicates a low reliability of the questionnaire. In our study, the number of cases, alpha and the number of items are 27, 0.7524 and 12 , respectively. The value of alpha equal to 0.75 percent shows a high reliability of the questionnaire

\subsection{Data Analysis Method}

Chi-square test, Friedman test, confidence intervals for means, Wilcoxon signed-rank test, binominal test, and Student's t-test are used at the confidence level of 95 percent to test the hypotheses of this research.

\subsection{General Research Method}

The general studies reveal that, 87 percent of the respondents are the graduates of the field of study accounting, from which $60 \%$ hold bachelor's degree and the remaining $40 \%$ higher degrees. Thirtyfive percent of the respondents were chief auditors, $47 \%$ senior auditors, and $18 \%$ managers. Seventy-five percent of the respondents had more than four years experience. Eighty-seven percent of the respondents had stock transactions in their records, and seventy-nine percent of the total 
respondents were more than three years in the capital market. According to the above facts, it can be concluded that most respondents were sufficiently qualified in terms of expertise and profession to answer the questions of the questionnaire.

\section{The results}

\subsection{The First Hypothesis}

Ratio or binomial test has been applied to test the first hypothesis stating that, unqualified audit reports have positive effects on stock price. Together with the above-mentioned statistical test for the evaluation of the effects, as there are more than two choices, chi-square test, Student's t-test, and confidence interval for mean are used. It must be noted that the results of the statistic methods confirmed each other.

\section{The Results of the Binominal Test of the first Hypothesis}

The statistical hypotheses $\mathrm{H}_{1}$ and $\mathrm{H}_{0}$ are as follows:

$\mathrm{H}_{0}$ : the ratio of the respondents who gave a positive answer to the positive effects of unqualified audit opinion on stock price is less than or equal to half of the respondents.

$\mathrm{H}_{1}$ : the ratio of the respondents who gave a positive answer to the positive effects of unqualified audit opinion on stock price is more than half of the respondents.

$\mathrm{Z}$ statistic equal to 5.4 is considerably greater than critical value, which is equal to 1.64 . Therefore, $\mathrm{H}_{0}$ is rejected.

\section{Chi-Square Test}

If a variable is measured by classification, the main question is what the ratio of answer to each class. For example, when the answer to the question of the effects of unqualified opinion on the stock return is classified into very high, high, moderate, low, and very low, the main purpose is to determine whether the rate of answers given to the classes of high and very high is more than the rate of answers given to the classes of low and very low.

The statistical hypotheses $\mathrm{H}_{0}$ and $\mathrm{H}_{1}$ are as follows:

$\mathrm{H}_{0}$ : the ratio of answers given to the different classes is identical.

$\mathrm{H}_{1}$ : the ratio of answers is different at least in two classes.

$\chi^{2}=30.870$, the degree of freedom $=4$ and the level of significance is equal to 0.000 .

Since the value of the level of significance is lower than 5 percent, $\mathrm{H}_{0}$ is rejected. The study of the collected data shows that 43 percent of the respondents selected the option moderate, and 37 percent of them selected the classes lower than moderate.

\section{Confidence Interval for Mean:}

The confidence interval of $95 \%$ for mean is expressed as follows:

$$
\left(\bar{X}-1.96 \frac{S}{\sqrt{n}}, \bar{X}+1.96 \frac{S}{\sqrt{n}}\right),
$$


where $\bar{X}$ stands for mean, s for standard deviation, and $n$ is the number of observation. The mean interval is equal to 2.5 and 2.98, respectively indicating that the answers are focused on the options moderate and low. The result of this test confirms the result of chi-square test.

Student's t-test:

Student's t-test is applied for the comparison of a variable or sample with a constant.

The statistical hypotheses $\mathrm{H}_{0}$ and $\mathrm{H}_{1}$ are as follows:

$\mathrm{H}_{0}: \mu=3$

$\mathrm{H}_{1}: \mu \neq 3$

The test statistic is defined as follows:

$$
t=\frac{\bar{X}-\mu_{0}}{\frac{S}{\sqrt{n}}}=-2.133
$$

Considering the test statistic, the significance level of $36 \%$, and the value of $t, \mathrm{H}_{0}$ is rejected. Considering that $t$ value is negative, $\mu \leq 3$. As shown, the result of this test confirms the results of chisquare and confidence interval for mean. This means that most respondents believe that unqualified audit report has a positive effect on stock return at low to moderate level.

\subsection{The second hypothesis}

To test the second hypothesis, the following statistical methods have been used.

\section{Ratio Test for the Second Hypothesis}

The statistical hypotheses $\mathrm{H}_{0}$ and $\mathrm{H}_{1}$ are as follows:

$\mathrm{H}_{0}$ : the ratio of the respondents who gave a positive answer to the negative effects of adverse audit opinion on stock price is less than or equal to half of the respondents.

$\mathrm{H}_{1}$ : the ratio of the respondents who gave a positive answer to the negative effects of adverse audit opinion on stock price is more than half of the respondents.

$\mathrm{Z}$ statistic is equal to 6.6 and it is considerably greater than critical value, 1.64 . As most respondents $(83 \%)$ believe that adverse audit opinion has a negative effect on the stock return of companies. Therefore, the second hypothesis is confirmed.

\section{Chi-Square Test}

The statistical hypotheses $\mathrm{H}_{0}$ and $\mathrm{H}_{1}$ are as follows:

$\mathrm{H}_{0}$ : the ratio of answers given to the different classes is identical.

$\mathrm{H}_{1}$ : the ratio of answers is different at least in two classes.

$\chi^{2}=28.506$, the degree of freedom is equal to 4 and the level of significance equal to 0.000 .

As the value of the level of significance is lower than 5 percent, therefore $\mathrm{H}_{0}$ is rejected. The study of the collected data shows that 68 percent of the respondents selected the options higher than moderate.

\section{Confidence Interval for Mean:}

The result of this test confirms the result of above chi-square test, and therefore, $\mathrm{H}_{0}$ is rejected. The mean interval is 3.53 and 4.6, indicating that the answers are focused on the options high and very high. 
Student's t-test:

The statistical hypotheses $\mathrm{H}_{0}$ and $\mathrm{H}_{1}$ are as follows:

$\mathrm{H}_{0}: \mu=3$

$\mathrm{H}_{1}: \mu \neq 3$

The test statistic is defined as follows:

$t=\frac{\bar{X}-\mu_{0}}{\frac{S}{\sqrt{n}}}=6.05$

Considering the test statistic, the significance level of 0.000 , and the value of $t, \mathrm{H}_{0}$ is rejected. Since $\mathrm{t}$ value is positive, therefore $\mu \geq 3$. As shown, the result of this test confirms the results of chi-square and confidence interval for mean. This means that most respondents believe that adverse audit report has a negative effect on stock return at the levels higher than moderate.

\subsection{The Third Hypothesis}

To test the third hypothesis, the following statistical methods have been used.

Ratio Test for the third Hypothesis

The statistical hypotheses $\mathrm{H}_{0}$ and $\mathrm{H}_{1}$ are as follows:

$\mathrm{H}_{0}$ : The ratio of the respondents who gave a positive answer to the negative effects of disclaimer audit opinion on stock price is less than or equal to half of the respondents.

$\mathrm{H}_{1}$ : The ratio of the respondents who gave a positive answer to the negative effects of disclaimer audit opinion on stock price is more than half of the respondents.

$\mathrm{Z}$ statistic is equal to 5.2 and it is considerably greater than critical value, which is equal to 1.64. Therefore, the null hypothesis is rejected. As most respondents $(76 \%)$ believe that disclaimer of audit opinion has a negative effect on the stock return of companies, the third hypothesis is confirmed.

\section{Chi-Square Test}

The statistical hypotheses $\mathrm{H}_{0}$ and $\mathrm{H}_{1}$ are as follows:

$\mathrm{H}_{0}$ : the ratio of answers given to the different classes is identical.

$\mathrm{H}_{1}$ : the ratio of answers is different at least in two classes.

$\chi^{2}=15.523$, the degree of freedom is equal to 4 , and the level of significance equal to 0.004 .

As the value of the level of significance is lower than 5 percent, therefore $\mathrm{H}_{0}$ is rejected. The study of the collected data shows that the respondents focus mostly on the options high and moderate more than other options.

\section{Confidence Interval for Mean:}

The result of this test confirms the result of above chi-square test. The mean interval is 3.52 and 3.01, indicating that the answers are focused on the options high and moderate.

Student's t-test:

The statistical hypotheses $\mathrm{H}_{0}$ and $\mathrm{H}_{1}$ are as follows:

$\mathrm{H}_{0}: \mu=3$

$\mathrm{H}_{1}: \mu \neq 3$ 
The test statistic is defined as follows:

$t=\frac{\bar{X}-\mu_{0}}{\frac{S}{\sqrt{n}}}=2.046$

Considering the $t$ statistic is equal to 2.046, and the significance level of $0.044, \mathrm{H}_{0}$ is rejected. Since $t$ value is positive, therefore $\mu \geq 3$. As shown, the result of this test confirms the results of chi-square and confidence interval for mean. This means that most respondents believe that disclaimer of audit report has a negative effect on stock return at the moderate and high levels.

\subsection{The Fourth Hypothesis}

To test the fourth hypothesis, binomial, Friedman, and Wilcoxon tests are used. Binomial test is applied to test the effectiveness of the above qualifications on stock return. Friedman test is used to determine whether the means of the rank of effects of the above-mentioned qualifications on stock return are identical or not, and finally Wilcoxon test is used for ranking the effectiveness of the qualifications in stock returns.

\section{Binomial Test}

The statistical hypotheses $\mathrm{H}_{0}$ and $\mathrm{H}_{1}$ are defined as follows:

$\mathrm{H}_{0}$ : The ratio of the respondents, who gave a negative answer to the effectiveness of the audit qualifications on stock return, is equal to half of the total respondents.

$\mathrm{H}_{1}$ : The ratio of the respondents, who gave a negative answer to the effectiveness of the audit qualifications on stock return, is not equal to half of the total respondents.

The test static of the qualifications leading to the modification of loss and gain statement is calculated as follows:

$$
Z=\frac{P-P_{0}}{\sqrt{\frac{P_{0}\left(1-P_{0}\right)}{n}}}=6.6
$$

The test static of the qualifications leading to the modification of balance sheet is $Z=-2.6$ and the test static of the qualifications leading to the modification of statement is $Z=7.4$. Considering the calculated statistic, $\mathrm{H}_{0}$ is rejected for all audit qualifications studied in this research.

Since the statistic of the qualifications, leading to the modification of the items of balance sheet, is negative and as the statistic of other qualifications is positive, it can be concluded that more than half of the respondents believe that the qualifications leading to the modification of the items of statement and those ones leading to the modification of the items in the both statement and balance sheet are effective in the stock return of companies. Moreover, less than half of the respondents believe that the qualifications leading to the modification of the items in the balance sheet are effective in the stock return of the companies.

\subsection{Friedman Test}

The statistic hypotheses $\mathrm{H}_{0}$ and $\mathrm{H}_{1}$ are defined as follows:

$\mathrm{H}_{0}$ : The mean ranks of the effects of the qualifications leading to the modification of the items of loss and gain statement, balance sheet, and both of them, are identical.

$\mathrm{H}_{1}$ : The mean ranks of the effects of the qualifications leading to the modification of the items of loss and gain statement, balance sheet, and both of them, are not identical. 
Considering the fact that the significance level is less than five percent, $\mathrm{H}_{0}$ is rejected $(17 \% \leq 5 \%)$. The mean ranks of the qualifications leading to the modification of the items of loss and gain statement, balance sheet, and both of them are 2.33, 1.61, and 2.06 respectively, and as shown, they are not equal to each other.

\subsection{Wilcoxon Test}

To compare the questions of this research in paired form, Wilcoxon test is used. For this purpose, the qualifications in question are classified into three groups and prioritized.

The statistical hypotheses $\mathrm{H}_{0}$ and $\mathrm{H}_{1}$ are defined as follows:

$\mathrm{V}_{1}=$ the effect of the qualifications leading to the modification of the items of loss and gain.

$\mathrm{V}_{2}=$ the effect of the qualifications leading to the modification of the items of balance sheet.

$\mathrm{V}_{3}=$ the effect of the qualifications leading to the modification of the items of both balance sheet and loss and gain statement.

\section{The Hypotheses of the Group A}

$\mathrm{H}_{0}$ : the mean ranks of $\mathrm{V}_{1}$ and $\mathrm{V}_{2}$ on stock return are identical.

$\mathrm{H}_{1}$ : the mean ranks of $\mathrm{V}_{1}$ and $\mathrm{V}_{2}$ on stock return are not identical.

\section{The Hypotheses of the Group B}

$\mathrm{H}_{0}$ : the mean ranks of $\mathrm{V}_{1}$ and $\mathrm{V}_{3}$ on stock return are identical.

$\mathrm{H}_{1}$ : the mean ranks of $\mathrm{V}_{1}$ and $\mathrm{V}_{3}$ on stock return are not identical.

\section{The Hypotheses of the Group C}

$\mathrm{H}_{0}$ : the mean ranks of $\mathrm{V}_{2}$ and $\mathrm{V}_{3}$ on stock return are identical.

$\mathrm{H}_{1}$ : the mean ranks of $\mathrm{V}_{2}$ and $\mathrm{V}_{3}$ on stock return are not identical.

The significance levels of the hypotheses of group A, group B, and group C are equal to $0.002,0.623$, and 0.005 , respectively. Since the significance level of the hypotheses of the group A and $\mathrm{C}$ are smaller than $5 \%$, therefore $\mathrm{H}_{0}$ is rejected. In contrast, as the significance level of the hypotheses of the group $\mathrm{B}$ is greater than $5 \%$, therefore $\mathrm{H}_{0}$ is confirmed. According to the statistic results obtained from the above-mentioned tests, the fourth hypothesis of this research is confirmed too. That means the qualifications leading to the modification of the items of statement and balance sheet, and both of them have more significant effects on the stock return than the qualifications leading to the modification of the items of balance sheet do.

\section{Concluding remarks}

One of the benefits of scientific researches is the documentation of the beliefs of experts working in a special field of study, and this converts an opinion or belief into a scientific theory. The issue that audit opinions contain valuable information for investors has been subject in many studies. In this research, we tested and analyzed this claim to use the results at higher level of confidence.

The findings of the tests of the hypotheses are as follows:

1- The test results of the first hypothesis on the positive effects of unqualified audit opinion on the stock return of companies confirmed the hypothesis. Most respondents selected the options 
moderate and low for this hypothesis. That means most respondents believe that unqualified auditor's report has a positive effect on the stock return of the companies at a level between low and moderate.

2- The test results of the second hypothesis on the negative effects of adverse audit opinion on the stock return of companies confirmed the hypothesis. Most respondents selected the options moderate and high for this hypothesis. That means most respondents believe that adverse auditor's report has a negative effect on the stock return of the companies at a level between moderate and high.

3- The test results of the third hypothesis on the negative effects of disclaimer of audit opinion on the stock return of companies confirmed the hypothesis. Like the second hypothesis, most respondents believe that disclaimer of auditor's report has a negative effect on the stock return of the companies at a level between moderate and high.

4- The test results of the fourth hypothesis on the higher effectiveness of the qualifications leading to the modification of the items of statement in comparison to that of the qualifications leading to the modification of the items of the balance sheet, confirmed the hypothesis. More than half of the respondents believe that the qualifications leading to the modification of the items of loss and gain have more effects on the stock returns of companies than the qualifications leading to the modification of balance sheet items do.

Based on the results, we can conclude that at a confidence level of $95 \%$ there is a significant relationship between auditor's reports and return on stock. Auditing thus is regarded as a process that produces information that can be used suitably for judgment, and it can confirm or reject the value of economic information obtained by accounting processes to increase or decrease accordingly the value of such information. As mentioned in the chapter of review of literature, the studies conducted on the effects of the informative content of auditor's report on financial factors such as stock price and return confirm that these factors are affected by the content of auditor's reports, and this is consistent with the results of this research.

\section{References}

Chen, K., \& Church, B. (1992). Going-concern opinions and the market's reaction to bankruptcy filings. The Accounting Review, 61, 249-262.

Chen, K., \& B. Church. (1996). Default on debt obligations and the issuance of going-concern opinions. The Accounting Review, 71, 117-128.

Davis, R. (1982). An empirical evaluation of auditors' "subject-to" opinions. Auditing: A Journal of Practice and Theory, 2(1), 13-32.

Dopuch, N., R. Holthausen \& R. Leftwich (1986). Abnormal stock returns associated with media disclosures of 'subject to' qualified audit opinions. Journal of Accounting and Economics, 8(2), 91-172.

Elliott, J. (1982). Subject to audit opinions and abnormal security returns - outcomes and ambiguities. Journal of Accounting Research, 20(2), 617-638.

Hajiha, Z. (2012). Application of Delphi method for determining the affecting factors upon audit risk model. Management Science Letters, 2(1), 379-390.

Khatami, M.A. (1997). The Effects of Audit Qualifications on the Stock Price and Analysis of the Annual Financial Statements by the Brokers of Tehran Stock Exchange. Unpublished PhD Thesis of Accounting Submitted to the Science and Research Branch of the Islamic Azad University of Tehran, 34-35.

Loudder, M., Khurana, I., Sawyers, R., Cordery, C., Johnson, C., Lowe, J., \& Wunderle, R. (1992). The information content of audit qualifications. Auditing: A Journal of Practice and Theory, 11(1), 69-82. 